\title{
Young red supergiants and the near infrared light appearance of disk galaxies
}

\author{
James E. Rhoads ${ }^{1}$ \\ Princeton University Observatory, Peyton Hall, Princeton, NJ 08544 \\ I: jrhoads@noao.edu
}

\begin{abstract}
Disk galaxies often show prominent nonaxisymmetric features at nearinfrared wavelengths. Such features may indicate variations in the surface density of stellar mass, contributions from young red supergiants in star forming regions, or substantial dust obscuration. To distinguish among these possibilities, we have searched for spatial variations in the $2.3 \mu \mathrm{m}$ photometric CO index within the disks of three nearby galaxies (NGC 278, NGC 2649, \& NGC 5713). This index measures the strength of the absorption bands of molecular CO in stellar atmospheres, and is strong in cool, low surface-gravity stars, reaching the largest values for red supergiants.

We observe significant spatial $\mathrm{CO}$ index variations in two galaxies (NGC 278 \& NGC 5713), indicating that the dominant stellar population in the near-infrared is not everywhere the same. Central CO index peaks are present in two galaxies; these could be due to either metallicity gradients or recent star formation activity. In addition, significant azimuthal CO index variations are seen in NGC 278. Because strong azimuthal metallicity gradients are physically implausible in disk galaxies, these features are most naturally explained by the presence of a young stellar population.

The fraction of $2 \mu \mathrm{m}$ light due to young stellar populations in star forming regions can be calculated from our data. Overall, young stellar populations can contribute $\sim 3 \%$ of a (normal) galaxy's near infrared flux, which is consistent with other global properties (e.g., the near-IR Tully-Fisher relation). Locally, this fraction may rise to $\sim 33 \%$. Thus, young stars do not dominate the total near infrared flux, but can be locally dominant in star forming regions, and can bias estimates of spiral arm amplitude or other nonaxisymmetric structures in galaxies' mass distributions.
\end{abstract}

Subject headings: galaxies: photometry, galaxies: stellar content, infrared: galaxies

\footnotetext{
${ }^{1}$ Present address: Kitt Peak National Observatory, P.O. Box 26732, Tucson, AZ 85726-6732
} 


\section{Introduction}

In the search for reliable tracers of stellar mass, near infrared light has become a popular choice. Astrophysical arguments in its favor are the relative insensitivity of near infrared light to hot, young stars, and the relatively small obscuration by dust at these wavelengths. The recent development of near infrared array detectors has opened the way for surface photometry at $1-3.5 \mu \mathrm{m}$ wavelengths, allowing practical studies of galaxies' structure in this wavelength range.

Studies of disk galaxies have shown that spiral arms and other nonaxisymmetric features frequently have large amplitudes - of order 2:1 arm to interarm contrast or more (Rix \& Rieke 1993, Rix \& Zaritsky 1995, Terndrup et al 1994). Spiral arms are prominent at blue wavelengths, where hot, luminous OB stars emit much of their radiation. Blue light can thus be said to trace sites of star formation. Because near infrared (NIR) light in $\mathrm{H}(1.6 \mu \mathrm{m})$ and $\mathrm{K}(2.2 \mu \mathrm{m})$ bands is in the Rayleigh-Jeans part of the spectrum for even very cool stellar photospheres, the contributions of hot stars there are minimized and the longer-lived, cooler stars are well represented. NIR light is also less attenuated by interstellar dust than is optical light, which will make arms appear smoother at $2 \mu m$ than at optical wavelengths.

If a galaxy formed most of its stars more than a rotation period $\left(\sim 10^{8}\right.$ years $)$ ago, then the older stellar populations have had time to orbit the galaxy, and will no longer reflect the spatial distribution of star formation regions. Assuming these old stars dominate the near infrared (NIR) light, this light will reflect the underlying stellar mass distribution. If it is strongly concentrated to spiral arms, large arm amplitudes must then be explained by stellar dynamics alone. This can be restated by noting that if NIR light is dominated by old stars, then NIR light approximately obeys a continuity equation. Nonaxisymmetric features a factor $\alpha$ brighter than the background disk then imply velocity perturbations of order $(\alpha-1) / \alpha \times\left(v_{c}-\Omega_{p} R\right)$, where $v_{c}$ is the circular speed, $\Omega_{p}$ the angular pattern speed, and $R$ the galactocentric distance. For a 2:1 arm to interarm ratio $(\alpha=2)$ and regions of the galactic disk away from corotation resonance (where we expect $\left|v_{c}-\Omega_{p} R\right| /\left|v_{c}\right| \sim 0.5$ ), we obtain velocity perturbations $\sim v_{c} / 4$.

However, the contribution of young stars to NIR light is not zero. In fact, it can greatly exceed the contribution from the upper main sequence alone, because some fraction of high mass stars pass through a luminous red supergiant phase at the ends of their lives. These stars are much younger than typical disk galaxy rotation periods (age $\sim 10^{7}$ years [e.g., Schaller et al 1992]), and so will still trace the stellar birthplaces with reasonable fidelity. If they contribute a substantial fraction of the near infrared light from a galaxy, the advantages of NIR as a measure of stellar mass distribution may be but little. In this 
work, we attempt to directly determine how much young supergiant stars contribute to features in NIR images of disk galaxies, and hence how well the stellar mass distribution of disk galaxies can be inferred from NIR surface photometry.

Determining the amount of supergiant light in the near-infrared has several applications. First, orbit synthesis models for the mass distribution and dynamics of disk galaxies often attempt to reproduce a surface mass density proportional to NIR surface brightness (Athanassoula 1996, Quillen 1996, Grosbøl \& Patsis 1996). Replacing this direct proportionality with something more accurate would be beneficial for such models.

Second, population synthesis models that seek to predict the spectra of composite stellar populations may benefit if substantially improved constraints on the numbers of supergiants become available.

Third, theoretical models of the late evolution of massive stars are highly uncertain, primarily because of difficulties modeling mass loss, convective overshooting, and semiconvection (e.g. Langer \& Maeder 1995). The factors controlling whether a particular star will or will not become a red supergiant are thus ill-understood. However, different models will lead to different predictions for the numbers of red supergiants, and by studying the contribution of red supergiants to near-IR light we may provide an observational check on stellar evolution theory.

In the following sections, we will discuss our chosen tool, the CO index (section II); describe the observations (section III) and analysis (section IV); present our results (section V); and discuss the implications of our findings (section VI).

\section{2. $\quad 2.3 \mu m \mathrm{CO}$ absorption as a diagnostic tool}

The $2.3 \mu \mathrm{m}$ absorption due to $\mathrm{CO}$ molecules in the atmospheres of cool stars provides a useful tool for the study of stellar populations at near infrared wavelengths. The strength of this absorption is traditionally measured using the CO index, which is essentially a photometric color defined between intermediate bandpass filters at wavelengths of $2.21 \mu \mathrm{m}$ and $2.34 \mu \mathrm{m}$. Specifically, $\mathrm{CO}=2.5 \log _{10}\left(F_{2.21} / F_{2.34}\right)+q$, so that larger $\mathrm{CO}$ indices indicate stronger absorption features. The constant $q$ is adjusted so that stars with no CO absorption have index $\approx 0$.

The CO index increases strongly as surface gravity decreases, which makes it an effective diagnostic of stellar luminosity class (Baldwin, Frogel, \& Persson 1973). It also increases with increasing stellar metallicity for giant stars of (approximately) fixed surface 
temperature (measured by $V-K$ color) (Frogel, Cohen, \& Persson 1983), and increases with decreasing surface temperature (measured by $J-K^{\prime}$ color) for Galactic giant stars (McWilliam \& Lambert 1984). Note that several different filter sets have been used to define CO indices in the literature (e.g. Baldwin et al 1973, Frogel et al 1978, McWilliam \& Lambert 1984), and additional definitions have been used to characterize spectroscopically measured CO absorption (e.g. Lançon, Rocca-Volmerange, \& Thuan 1996). This can complicate comparison of CO indices from different sources. We have set the zero point of our CO indices using the standard star list of Elias et al (1982), who use the filter system defined by Frogel et al (1978). The CO index of Vega is set to 0 in this system. The central wavelengths and bandpasses of our continuum filter $(2.21 \mu \mathrm{m}, 0.10 \mu \mathrm{m})$ and CO band filter $(2.34 \mu \mathrm{m}, 0.09 \mu \mathrm{m})$ are similar to those used by Frogel et al $(1978)(2.20 \mu \mathrm{m}, 0.11 \mu \mathrm{m}$; $2.36 \mu \mathrm{m}, 0.08 \mu \mathrm{m})$. Thus, our indices should reproduce the system of Frogel et al (1978) fairly well.

The behavior of the CO index for stellar atmosphere models of differing metallicity, surface temperature, and surface gravity has been explored in detail by Bell \& Briley (1991). The dependences on metallicity and surface temperature follow naïve intuition well: In order for $\mathrm{CO}$ molecules to be abundant, there must be adequate supplies of carbon and oxygen, and the temperature must not be so high that the molecules dissociate. Thus the $\mathrm{CO}$ index decreases with decreasing metallicity or increasing surface temperature. The dependence on surface gravity is less obvious. As pressure increases, the opacity in the $\mathrm{CO}$ absorption band rises, but the continuum opacity rises faster. The net effect is that the equivalent width of the absorption bands rises for stars of lower surface gravity (Bell \& Tripicco 1991; Bell \& Briley 1991). Frogel (private communication) points out that microturbulence increases with decreasing surface gravity, and so offers another mechanism for the luminosity dependence: As the microturbulent velocity rises, saturated CO lines are broadened and their equivalent widths rise.

Because the index measures the continuum only on the blue side of the absorption feature, it is affected by changes in the continuum slope even in the absence of $\mathrm{CO}$ absorption. For pure black body spectra, $C O \approx-0.07(J-K) \approx-0.15(H-K)$. Thus in cool stars zero index does not exactly correspond to no absorption.

If the CO index shows spatial variations in a galaxy, it implies a spatial variation of the underlying stellar population. In general, this will also imply a variation in the near infrared light to mass ratio. Because differential rotation causes phase mixing of the stars in disk galaxies on timescales of a few rotation periods (i.e., a few $\times 10^{8}$ years), while chemical evolution proceeds over the star forming lifetime of the galaxy (i.e., $\sim 10^{10}$ years for large disk galaxies), no substantial azimuthal variation in metallicity is expected. Azimuthal 
variations in the $\mathrm{CO}$ index are therefore most naturally explained by star formation activity $\lesssim 10^{8}$ years ago.

\section{Observations}

\subsection{Sample selection}

The galaxies in our sample were face-on disk galaxies chosen to span a range of spiral arm morphologies and star formation activity. In addition, they had to be small enough to fit within the $2^{\prime}$ field of the instrument (described below), and to lie in a suitable part of the sky for observation from Apache Point Observatory (latitude $32^{\circ} 46^{\prime} 49.3^{\prime \prime} \mathrm{N}$ ).

The sample consists of three galaxies. The first of these, NGC 278, was chosen because its optical image (Wray 1988; Sandage \& Bedke 1994) shows prominent bright patches in its inner regions. These are presumably areas of active star formation, making NGC 278 a good place to look for the signature of young stars in near infrared light. It also has a very high central surface brightness $\left(\mu_{K} \approx 14.1 \mathrm{mag} / \operatorname{arcsec}^{2}\right.$ in $1.3^{\prime \prime}$ seeing). It lies at the lowest redshift in the sample (heliocentric velocity $641 \mathrm{~km} / \mathrm{s}$ [de Vaucouleurs et al 1991]), meaning that its distance is the least certain (with perhaps $50 \%$ errors possible due to peculiar velocity).

The second galaxy, NGC 2649, is a grand design spiral with smooth arms of moderate amplitude. No nonstellar objects (besides the galaxy itself) are apparent in our images of NGC 2649, from which we conclude that it has no massive satellites $\left(M \gtrsim 5 \times 10^{8} M_{\odot} \approx 0.003 M(\mathrm{NGC} 2649)\right)$ of normal mass-to-light ratio within $17 \mathrm{kpc}$. The Palomar Observatory Sky Survey (POSS) plates allow this limit to be extended to at least $130 \mathrm{kpc}$ at a slightly less stringent limit (no satellite with $M \gg 0.02 M(\mathrm{NGC} 2649)$ ).

Finally, the third galaxy, NGC 5713, has an unusual morphology that suggests it has recently been disturbed, perhaps by an interaction with another galaxy. NGC 5713 has a large FIR to optical luminosity ratio (twice that of NGC 278 and six times that of NGC 2649), suggesting a large mean star formation rate.

Properties of these galaxies derived from the literature and from our photometry are summarized in Table I. 


\begin{tabular}{|l|ccc|}
\hline Galaxy & NGC 2649 & NGC 278 & NGC 5713 \\
\hline RA $(2000.0)$ & $8: 44: 06.0$ & $0: 52: 01.6$ & $14: 40: 11.52$ \\
Dec $(2000.0)$ & $34: 43: 01.6$ & $47: 33: 18$ & $-0: 17: 25.8$ \\
$V_{\odot}(\mathrm{km} / \mathrm{s})^{\mathrm{a}}$ & $4244 \pm 6$ & $641 \pm 9$ & $1883 \pm 4$ \\
$V_{l g}(\mathrm{~km} / \mathrm{s})^{\mathrm{b}}$ & $4249 \pm 6$ & $931 \pm 9$ & $1777 \pm 4$ \\
$d(\mathrm{Mpc})^{\mathrm{c}}$ & 57 & 12.4 & 24 \\
$m_{F I R}-B_{T}^{0 \mathrm{~d}}$ & 0.36 & -1.02 & -1.71 \\
$m_{21}^{0}-B_{T}^{0 \mathrm{e}}$ & 2.82 & 2.87 & 1.47 \\
$K^{\prime \mathrm{f}}$ & 10.11 & 7.73 & 8.43 \\
$J^{\mathrm{f}}$ & & & 9.50 \\
$B_{T}^{0}-K^{\prime}$ & 2.71 & 2.86 & 3.08 \\
$r_{e x p}(2.22 \mu m)^{\mathrm{g}}$ & $15.6^{\prime \prime} \pm 1.5$ & $11.7^{\prime \prime} \pm 0.6$ & $14^{\prime \prime} \pm 1$ \\
\hline
\end{tabular}

${ }^{a}$ Heliocentric velocity from de Vaucouleurs et al 1991 [RC3].

${ }^{b}$ Velocity relative to the local group, determined using a correction formula supplied by M. Strauss.

${ }^{c}$ Calculated as $d=V_{l g} / H_{0}$, assuming $H_{0}=75 \mathrm{~km} / \mathrm{s} / \mathrm{Mpc}$.

${ }^{d}$ Far infrared to extinction- and inclination-corrected blue light ratio, in magnitude units, from RC3.

${ }^{e}$ Corrected $21 \mathrm{~cm}$ line flux to blue light ratio, in magnitude units, from RC3.

${ }^{f}$ Total near-infrared magnitudes, derived from Apache Point surface photometry. Estimated $1 \sigma$ errors are 0.05 mag. No corrections for extinction or inclination have been applied.

${ }^{g}$ Exponential scale length based on $2.22 \mu m$ maps. 


\subsection{Observations}

All observations were taken at the Astrophysical Research Consortium (ARC) 3.5 meter telescope at Apache Point Observatory 2], New Mexico, USA, using the facility near-infrared camera, GRIM II (built by M. Hereld, B. Rauscher, and collaborators).

The data were taken and reduced following standard practice for near infrared photometry. Additionally, the data were processed independently through a model fitting technique that allows better control of certain systematic errors; the results of the two methods were consistent. Further details of the observations are given in the remainder of this section. The data analysis procedures are described in the following section.

We used three filters: A broad $K^{\prime}$ band filter (Wainscoat \& Cowie 1992) with central wavelength $2.114 \mu \mathrm{m}$ and $0.343 \mu \mathrm{m}$ full width at half maximum (FWHM); a narrow continuum filter with transmission-weighted mean wavelength $2.210 \mu \mathrm{m}$ and FWHM $0.100 \mu m(4.5 \%)$; and a narrow CO band filter with mean wavelength $2.34 \mu m$ and FWHM $0.091 \mu m(3.9 \%)$. (Quoted filter characteristics are based on filter traces made by the manufacturer at the operating temperature for the filters.)

The data was taken over four nights, 1995 December 11 (NGC 278, NGC 2649), 1995 December 14 (NGC 2649), 1995 December 31 (NGC 278, NGC 2649), and 1996 April 29 (NGC 5713). Conditions were photometric on December 11 and on April 29, while some cirrus was present on the remaining two nights. Absolute calibration of the photometry used only data from the photometric nights. Seeing was typically $1-1.5^{\prime \prime}$ on December 11, 14, and 31, and was $\sim 4^{\prime \prime}$ on April 29.

On 1996 April 29, we obtained useful J, H, and $K^{\prime}$ band calibration data from three stars in the Elias et al (1982) list (HD 201941, HD 129655, and HD 136754). Four stars (BD+3 2954 , HD 161903, HD 201941, and HD 129655) were observed in the CO index filters. The airmass range covered was from 1.2 to 3.9. The resulting transformations from instrumental to standard magnitudes were $m_{K^{\prime}}=K-(22.354 \pm 0.014)+(0.079 \pm 0.006) X$ and $m_{2.34}-m_{2.21}=C O+(0.283 \pm 0.014)+(0.032 \pm 0.006) X$. Here $\mathrm{K}, \mathrm{J}$, and CO are tabulated standard magnitudes, $m_{K^{\prime}}, m_{2.34}$, and $m_{2.21}$ are instrumental magnitudes defined as $-2.5 \log _{10}(\mathrm{ADU} / \mathrm{s})$, and $X$ is the airmass of the observation.

On 1995 December 11, useful photometric calibration data was obtained in the CO index filters from G77-31 and HD 40335 (from the list by Elias et al [1982]); and in $K^{\prime}$

\footnotetext{
${ }^{2} \mathrm{APO}$ is privately owned and operated by the Astrophysical Research Consortium (ARC), consisting of the University of Chicago, Institute for Advanced Study, Johns Hopkins University, New Mexico State University, Princeton University, University of Washington, and Washington State University.
} 
band from Feige 11, SA94-242, and GD71 (from the UKIRT standards list by Casali \& Hawarden [1992]). The less extensive range of airmass (1.1 to 1.6) and color data allowed only zero points to be computed. These were $m_{K^{\prime}}=K-(22.191 \pm 0.007)$ and $m_{2.34}-m_{2.21}=C O+(0.373 \pm 0.010)$. The airmass range of the actual data on 1995 December 11 is small (1.05 to 1.35 ), so that uncorrected extinction terms are unlikely to exceed $0.02 \mathrm{mag}$ in $K^{\prime}$ band and $0.01 \mathrm{mag}$ in $\mathrm{CO}$ index.

Observations of each source consisted of a series of short (9-40 second) exposures of the object, interspersed with equal length exposures of nearby sky frames. These exposures were chosen to accumulate $\sim 5 \times 10^{4}$ electrons per pixel from the night sky, so that read noise ( $\sim 100$ electrons) is not a dominant source of error while the chip remains well below saturation thresholds $\left(\sim 13 \times 10^{4}\right.$ electrons per pixel). The telescope pointing was shifted randomly by a few arcseconds between exposures, so that a given point on the sky was sampled by many different pixels of the detector. To extract estimates of the target field's surface brightness distribution from individual object frames, we first subtracted the sky background using a combination of two to four sky frames taken immediately before and after the object frame. This step also removed most bias and dark current contribution to the signal. Because the bias structure of the chip showed small fluctuations from exposure to exposure and from line to line, the next step was to apply an additive correction to each line, ensuring that regions of the chip far from the galaxy have median pixel value zero. This step also has the effect of subtracting any residual sky signal, which may be important when the variable sky brightness is not adequately described by a linear trend in time. Note that the galaxies all had $2.21 \mu \mathrm{m}$ scale lengths $\leq 16^{\prime \prime}$, which is sufficiently small compared to the $121^{\prime \prime}$ chip that useful sky level measurements are possible at the edges of object frames.

The sky-subtracted data frames were then flat fielded, i.e., divided by a flat field image in order to correct for pixel-to-pixel variations in the detector's quantum efficiency and gain. We used "supersky" flat field images. These were constructed for each band and each night of observations by subtracting a mean dark current frame from a large number of sky frames, taking a pixel-by-pixel median of those frames, and normalizing the result to have median pixel value unity.

Finally, the individual data frames were registered and combined. Registration used astrometry of a bright, sharply peaked source (generally the nucleus of the galaxy, occasionally a nearby star) to determine appropriate fractional pixel shifts, which were then applied using bilinear interpolation. The shifted frames were then combined using a pixel-by-pixel median. The median ensures that bad pixels, cosmic ray hits, stars in the sky frames, and other artifacts in individual object frames have little effect on the final map, since such imperfections typically affect $\lesssim 1 \%$ of the pixels in a given data frame. 
The instrument response is mildly nonlinear. We have reduced the data first without and subsequently with nonlinearity corrections. The only significant change to the results is in the zero points of the photometry: $\mathrm{CO}$ indices are reduced by 0.05 in the linearized data, and total magnitudes of the galaxies change by $0.05-0.11$. Relative CO index measurements between different regions of the same galaxy were essentially unaffected. This is because the galaxies have a lower surface brightness than the night sky almost everywhere, so that the effect of the nonlinearity on sky-subtracted galaxy images is essentially to change the instrument gain by a few percent from image to image. We emphasize that our analysis is based primarily on azimuthal variations in the CO index, whose significance is unaffected by zero point shifts of the index.

An alternative data reduction method was used independently to produce images less sensitive to sky subtraction errors and bias structure variations in individual exposures. The method treats each pixel of the output image as one parameter of a linear model. Additional parameters representing bias level offsets in the data and offsets in the sky level of the image are included in a natural way. The model parameters yield a predicted data set through a linear map that can be determined using the flat field response of the chip and pointing information derived from astrometry of the individual exposures. The linear system is then solved for the model parameters using a regularized $\chi^{2}$ minimization (cf. Press et al 1992, sections 18.4-18.5). Similar techniques have been applied in the mid infrared by Van Buren \& Kong (1997). Our algorithm will be described in greater detail elsewhere.

Reduced $K^{\prime}$ band images of the galaxies are shown in figures $1 \mathrm{a}-1 \mathrm{c}$.

\section{Analysis}

The data analysis was done by defining masks that group pixels according to their surface brightness and location within the galaxy, and measuring the CO index of each pixel group. This method, which we call "masked aperture photometry," can combine the relatively high signal to noise ratios of traditional large-aperture photometry with the physical insight of a spatially resolved map.

Because we want to know what fraction of NIR light is contributed by young supergiants, we define masks according to the ratio of the measured surface brightness to a smooth axisymmetric model for the galaxy. In detail, if the local surface brightness is given by $f$ and the model by $m$, we define bright regions by $f / m-1>\tau_{1}$, and background

regions by $f / m-1<\tau_{2}$, with thresholds $\tau_{2}<\tau_{1}$. The choice of an appropriate model and 
threshold is an important step in the procedure. We considered using the axisymmetrized image of the galaxy as our model. However, this method will fail to flag the excess surface brightness from star formation in rings (like that in M31) or nuclei. We therefore chose to model the background light with a pure exponential disk. The exponential parameters were derived from a fit to the one-dimensional K' band profiles of the galaxies. The masks are shown in figures $1 \mathrm{~d}-1 \mathrm{f}$.

For any set $\mathcal{S}$ of pixels, we can define the total $\mathrm{CO}$ index of $\mathcal{S}$ in the obvious way: $\mathrm{CO}(\mathcal{S})=2.5 \log _{10}\left(\int_{\mathcal{S}} f_{2.21} d A / \int_{\mathcal{S}} f_{2.34} d A\right)+q$, where $f_{2.21}$ and $f_{2.34}$ are the local surface brightness in the $2.21 \mu \mathrm{m}$ and $2.34 \mu \mathrm{m}$ filters, $\int_{\mathcal{S}} d A$ represents the integral over the solid angle contained in $\mathcal{S}$, and $q$ sets the zero-point of the $\mathrm{CO}$ index scale.

Once the masks have been made, we can take their intersections with annuli about the galaxy center and form a curve of the $\mathrm{CO}$ index as a function of radius for bright and

background regions. This curve can be made either cumulative or differential. In this work we favor the differential version of the $\mathrm{CO}$ index- radius curves, since these have better spatial detail. The cumulative curves have higher total signal to noise ratio but offer less insight into where the data reliably measure differences in $\mathrm{CO}$ index.

\subsection{Error estimation}

The error in the measured $\mathrm{CO}$ index is composed of both random and systematic parts. These are of comparable importance in the data analysis.

Random errors are due to simple photon counting statistics. Because the surface brightness of our sample galaxies is generally well below that of the NIR night sky (reaching a peak of $1 / 3$ of sky on the nucleus of NGC 278), we estimated photon counting noise from the measured variance of sky pixels in our final images.

The most problematic systematic error is imperfect sky subtraction. The sky background is very bright (ranging from 12.8 to $13.4 \mathrm{mag} / \mathrm{arcsec}^{2}$ in $K^{\prime}$ in our data) and can change by $\sim 1 \%$ on timescales of a minute or so. Because it contains multiple components (night sky emission lines, thermal emission by the atmosphere, and thermal emission by the telescope and instrument), the flat field response may also vary slightly. To overcome this, it is necessary to take sky frames before and after object exposures and subtract a running median of the sky observations from each object frame. This eliminates most of the sky flux, and any residuals can be eliminated using a uniform value scaled by the flat field. Errors after this last step will be no worse than $23 \mathrm{mag} / \operatorname{arcsec}^{2}$. In practice, an additional complication was introduced by the fluctuations in bias level on the chip. Because bias level 
offsets are not proportional to the flat field, they cannot be handled exactly as sky level errors are. Distinguishing the amount of each error based on the outer regions of the chip is not always straightforward. In the worst case, the error introduced by handling a bias level offset as sky or vice versa is about 2 magnitudes worse than the above estimate, or up to $21 \mathrm{mag} / \operatorname{arcsec}^{2}$. These errors will vary in amplitude and sign from exposure to exposure, so should average out roughly as $1 / \sqrt{N_{\text {exp }}}$. They can also have some spatial structure, reflecting structure in the flat field.

We have used the variations in standard star fluxes measured at different places on the detector on 1996 April 29 to study possible residual flat fielding errors in our data. Standard star measurements that night were taken in an "X" pattern, i.e., one central measurement near the middle of the chip, and four more offset NE, NW, SE, and SW by $25^{\prime \prime}$ in right ascension and $25^{\prime \prime}$ in declination. We sky subtracted and flat fielded these data in the normal way, replaced bad pixels with the median of their neighbors, and measured standard star fluxes. We then compared the flux at each offset position with that at the central position. The mean magnitude offsets in the four quadrants were $-0.0073 \pm 0.0075$, $0.0066 \pm 0.0070,0.0027 \pm 0.0065$, and $0.0081 \pm 0.0091$. We repeated these calculations for the $\mathrm{CO}$ continuum filter alone and for the CO band filter alone, and found no evidence for systematic differences between the two filters. Thus, this test presents no compelling evidence for flat field errors, and rules out flat field errors much in excess of $1 \%$. Note also that such errors would affect only the sky-subtracted flux in the image; that is, a residual $1 \%$ flat field error here means $1 \%$ of the object surface brightness and not $1 \%$ of the sky level.

Consider now the effect of a spatially constant sky level error on the CO index measurement. For the interesting regime (where systematic errors are smaller than the mean surface brightness), the error $\delta \mathrm{CO}$ can be derived as follows:

$$
\begin{aligned}
& \mathrm{CO}+\delta \mathrm{CO}=2.5 \log _{10}\left(\frac{f_{2.21}+\delta f_{2.21}}{f_{2.34}+\delta f_{2.34}}\right)+q=2.5 \log _{10}\left(\frac{f_{2.21}\left(1+\delta f_{2.21} / f_{2.21}\right)}{f_{2.34}\left(1+\delta f_{2.34} / f_{2.34}\right)}\right)+q \\
& =2.5 \log _{10}\left(f_{2.21} / f_{2.34}\right)+q+2.5 \log _{10}\left(1+\delta f_{2.21} / f_{2.21}\right)-2.5 \log _{10}\left(1+\delta f_{2.34} / f_{2.34}\right)
\end{aligned}
$$

whence

$$
\begin{gathered}
\delta \mathrm{CO}=2.5 \log _{10}\left(1+\delta f_{2.21} / f_{2.21}\right)-2.5 \log _{10}\left(1+\delta f_{2.34} / f_{2.34}\right) \\
\approx 1.086\left(\delta f_{2.21} / f_{2.21}-\delta f_{2.34} / f_{2.34}\right) .
\end{gathered}
$$

Although the magnitude of this error is in general $\sim\left|\delta f_{2.21} / f_{2.21}\right| \sim\left|\delta f_{2.34} / f_{2.34}\right|$, it can be considerably smaller if $\delta f_{2.34} / \delta f_{2.21} \approx f_{2.34} / f_{2.21}$. Such a cancellation may be expected for some sky subtraction algorithms. For example, if the sky level is determined in an annulus around the center of a galaxy, the sky level errors will be proportional to the galaxy flux 
at some outer isophote. When such cancellation is present, we can define the quantity $\mathrm{CO}_{\delta}=2.5 \log _{10}\left(\delta f_{2.21} / \delta f_{2.34}\right)+q$ and derive the approximate relations

$$
\begin{gathered}
\delta \mathrm{CO} \approx 1.086\left(\frac{\delta f_{2.21}}{f_{2.21}}-\frac{\delta f_{2.34}}{f_{2.34}}\right)=\left(\frac{\delta f_{2.34}}{f_{2.34}}\right) \times 1.086\left(\frac{\delta f_{2.21}}{\delta f_{2.34}} \frac{f_{2.34}}{f_{2.21}}-1\right) \\
\approx\left(\frac{\delta f_{2.34}}{f_{2.34}}\right) \times 2.5 \log _{10}\left(\frac{\delta f_{2.21}}{\delta f_{2.34}} \frac{f_{2.34}}{f_{2.21}}\right)=\frac{\delta f_{2.34}}{f_{2.34}}\left(\mathrm{CO}_{\delta}-\mathrm{CO}\right) \approx \frac{\delta f_{2.21}}{f_{2.21}}\left(\mathrm{CO}_{\delta}-\mathrm{CO}\right)
\end{gathered}
$$

In our data set, we estimated the sky subtraction errors by examining exponential fits to the galaxies' radial profiles. The radial profiles from our data reduction procedure fall below the best fit exponentials at radii ranging from $\sim 2.25$ (for NGC 2649) to $\sim 5.5$ scale lengths (for NGC 278). By adding a suitably chosen constant, the light profile can be made to follow the exponential fit to the lowest measurable surface brightness in the data. This constant is taken as our estimate of the sky subtraction error in the individual filters. Using this estimator, we find $-0.5 \lesssim \mathrm{CO}_{\delta} \lesssim 0.3$ for our galaxies, and we therefore use the larger of $0.5\left(\delta f_{2.21} / f_{2.21}\right)$ and $0.5\left(\delta f_{2.34} / f_{2.34}\right)$ for $\delta \mathrm{CO}$. This assumes a modest cancellation of the sky subtraction errors in the two bands. This analysis suggests that sky subtraction errors in the CO index are $\sim \pm 0.01$ at $\mu_{K^{\prime}}=18 \mathrm{mag} / \operatorname{arcsec}^{2}$, with some differences from galaxy to galaxy. Note that the stellar disks of galaxies are believed to cut off at $\sim 4$ [optical] scale lengths (e.g., Robin, Crezé, \& Mohan 1992), so that the observed end of the exponential profile may be real in most of our sample. If so, our error estimates are conservative.

Because sky subtraction errors are largely a function of local surface brightness, the accuracy of a differential measurement of CO index between two regions at similar surface brightness can be much better than the above calculations suggest. Consider the quantity $\left(\mathrm{CO}_{1}-\mathrm{CO}_{2}\right)=2.5 \log _{10}\left[\left(f_{2.21,1} / f_{2.34,1}\right) \times\left(f_{2.34,2} / f_{2.21,2}\right)\right]$ when the surface brightnesses in regions 1 and 2 are comparable. In the ideal limit $f_{2.21,1}=f_{2.21,2}=f_{2.21}$, $\left(\mathrm{CO}_{1}-\mathrm{CO}_{2}\right)=2.5 \log _{10}\left(f_{2.34,2} / f_{2.34,1}\right) \approx 1.086\left[\left(f_{2.34,2}-f_{2.34,1}\right) / \overline{f_{2.34}}\right]$, which is clearly unaffected by errors in $f_{2.21}$. (Here $\left.\overline{f_{2.34}} \equiv\left(f_{2.34,1}+f_{2.34,2}\right) / 2 \approx f_{2.34,1} \approx f_{2.34,2}\right)$. Errors $\delta f_{2.34}$ affect it as $\delta\left(\mathrm{CO}_{1}-\mathrm{CO}_{2}\right) \approx 1.086\left(\delta f_{2.34} / f_{2.34,2}-\delta f_{2.34} / f_{2.34,1}\right) \approx$ $1.086 \delta f_{2.34}\left[\left(f_{2.34,1}-f_{2.34,2}\right) /{\overline{f_{2.34}}}^{2}\right] \approx-\left(\delta f_{2.34} / \overline{f_{2.34}}\right) \times\left(\mathrm{CO}_{1}-\mathrm{CO}_{2}\right)$. Thus a differential $\mathrm{CO}$ index measurement can be accurate to \pm 0.01 even in the presence of fractionally large sky subtraction errors. In practice, this means relative measurements are good at the $\delta\left(\mathrm{CO}_{1}-\mathrm{CO}_{2}\right)<0.01$ magnitude level down to $\mu_{K^{\prime}} \approx 19.5$ in our data, provided the regions under comparison are at similar $\mu_{K^{\prime}}$ and are subject to identical sky subtraction errors.

A second source of systematic error is seeing variations. If the point spread function (PSF) is appreciably broader in one band than another, artificial color gradients will be induced near strong peaks (especially point sources) in the image. The magnitude of this 
effect is easily estimated under the approximation of a Gaussian PSF. Consider a change in the breadth $\sigma$ of the Gaussian PSF by a factor $(1+\alpha)$ between two bands. Then the surface brightness ratio at the peak of a point source will change by a factor $(1+\alpha)^{2}$, which introduces a color shift of $5 \alpha / \ln (10)$ magnitudes in the limit of small $\alpha$. For an extended source, the effect is diminished. For a source with a Gaussian profile of intrinsic width $\eta$, a change from seeing sigma $\beta$ to $(1+\alpha) \beta$ introduces a color shift $5 \alpha / \ln (10) /\left[1+(\eta / \beta)^{2}\right]$.

To avoid such seeing-induced color shifts, we have matched the spatial resolution of the 2.21 and $2.34 \mu \mathrm{m}$ images for each galaxy. This was done by convolving the higher resolution image with a Gaussian kernel of appropriate width. For NGC 278 and NGC 2649, the kernel width was determined by comparing the widths of stars present in the mosaics. In NGC 5713, our field does not contain stars. We therefore measured the FWHM of the galaxy nucleus in each sky-subtracted exposure. For NGC 5713, the nucleus FWHM varied smoothly throughout the observations, with no obvious discontinuities when changing between 2.34 and $2.21 \mu \mathrm{m}$ filters (which was done four times in all). We therefore smoothed the $2.21 \mu \mathrm{m}$ image to match the observed width of the $2.34 \mu \mathrm{m}$ image.

Even after this smoothing, NGC 5713 and NGC 278 have significantly increased CO indices in their nuclei. Residual errors in PSF matching could be of order $\alpha \lesssim 0.02$, and the nuclei are not point sources $(\eta / \beta \approx 1)$, so that errors in the central indices are of order 0.02. We therefore conclude that these two galaxies show a real increase in $\mathrm{CO}$ absorption in their nuclei. Outside the galactic nuclei, where features are less sharply peaked and our masks cover larger solid angles, the $\mathrm{CO}$ indices did not change measurably when seeing corrections were applied.

A final systematic effect that must be considered is redshift. The CO absorption beyond $2.29 \mu \mathrm{m}$ is composed of many individual bands, with bandheads separated by about $0.03 \mu \mathrm{m}$ and a width $\sim 0.0075 \mu \mathrm{m}$ at half of the maximum absorption. The $2.34 \mu \mathrm{m}$ filter contains only a few such features, and the measured CO index may change appreciably as these features redshift through the edges of the bandpass. At the lowest redshifts, where the entire filter lies redward of the bandhead, these changes will be small and not necessarily monotonic in redshift. At somewhat higher redshifts $(1000 \mathrm{~km} / \mathrm{s} \lesssim c a \lesssim 11000 \mathrm{~km} / \mathrm{s})$, the $\mathrm{CO}$ absorption covers only part of the bandpass, and the correction becomes substantial. At redshifts $c z \gg 12000 \mathrm{~km} / \mathrm{s}$ the $\mathrm{CO}$ index no longer measures $\mathrm{CO}$ absorption at all. (These velocity ranges are quoted for the APO filter set.) To compensate for this effect, we have calculated synthetic CO indices for model stellar spectra (from Bell \& Briley 1991) kindly provided by R. Bell and redshifted to the recessional velocities of our sample galaxies. The correction is roughly proportional to the strength of the absorption, and was calculated for a range of rest frame $\mathrm{CO}$ indices. The effect of continuum slope on the $\mathrm{CO}$ index must be 
considered when calculating redshift corrections, because at $0.003 \lesssim z \lesssim 0.04$, the amount of absorption in the $\mathrm{CO}$ filter changes much more with redshift than the continuum slope does. Taking the $J-K$ color as indicative of continuum slope, we suggest a redshift correction equation of the form $\mathrm{CO}_{\mathrm{obs}}=\alpha(z)\left[\mathrm{CO}_{\text {true }}+\beta(J-K)\right]-\beta(J-K)$. Here $\beta \approx 0.07$ (based on measured colors for black body spectra of different temperatures), and $\alpha(z)$ is determined by integration over model spectra.

The approximate behavior of $\alpha$ can be modeled by pretending that the optical depth of CO absorption is zero for $\lambda<2.29 \mu \mathrm{m}$, constant for $\lambda>2.29 \mu \mathrm{m}$, and similarly that the filter transmission is uniform in $\lambda_{1}<\lambda<\lambda_{2}$ and zero elsewhere. Then $\alpha(z) \approx\left(\lambda_{2}-(1+z) 2.29 \mu m\right) /\left(\lambda_{2}-\lambda_{1}\right)$ for $\lambda_{1}<(1+z) 2.29 \mu m<\lambda_{2}$. Combining this approximation with the redshift correction formula above gives $\mathrm{CO}_{\text {true }}-\mathrm{CO}_{\text {obs }} \approx\left[(1+z) 2.29 \mu m-\lambda_{1}\right] /\left(\lambda_{2}-\lambda_{1}\right) \times\left[\mathrm{CO}_{\text {true }}+\beta(J-K)\right]$. We can compare this to Frogel et al's (1978) correction, $\mathrm{CO}_{\text {true }}-\mathrm{CO}_{\mathrm{obs}} \approx 4.8 z$, by inserting values appropriate to the old stellar systems they studied and their filter system $(J-K \approx 0.9$,

$\left.\mathrm{CO}_{\text {true }} \approx 0.15, \lambda_{1}=2.32 \mu m, \lambda_{2}=2.40 \mu m\right)$. This yields $\mathrm{CO}_{\text {true }}-\mathrm{CO}_{\text {obs }} \approx 6(z-0.013)$ for $0.013<z<0.048$, which is fairly good agreement given the crudeness of our approximation for $\alpha(z)$.

We have corrected our measured CO indices for redshift effects using the general formula above. The values of $\alpha$ obtained for our sample by convolving APO filters with model spectra are $\alpha(641 \mathrm{~km} / \mathrm{s})=0.957, \alpha(1883 \mathrm{~km} / \mathrm{s})=0.868$, and $\alpha(4244 \mathrm{~km} / \mathrm{s})=0.645$. We have measured $J-K=1.07$ for NGC 5713. For the other two galaxies, we use $J-K \approx 0.9$, which is typical for Sb and Sbc galaxies like these (Peletier \& Balcells 1996). We use $\beta=0.07$.

\section{Results and Interpretation}

The CO index (corrected for redshift effects) is plotted as a function of angular radius for all sample galaxies in figure 2. The degree of spatial variation in observed CO index differs substantially among galaxies in our sample. At one extreme, NGC 278 shows strong and statistically significant differences $(\Delta \mathrm{CO} \approx 0.07)$ between the $\mathrm{CO}$ indices of bright patches and of the background disk in the annulus $16^{\prime \prime}<r<22^{\prime \prime}$. In addition, it shows a $\mathrm{CO}$ index peak $(\mathrm{CO} \approx 0.14)$ in the nucleus. The feature at $r \approx 20^{\prime \prime}$ corresponds to a prominent ring of star forming regions in the galaxy. Beyond $25^{\prime \prime}$, there is no significant difference between the $\mathrm{CO}$ indices of different regions. There is a radial gradient in measured $\mathrm{CO}$ index, but this should be interpreted with extreme caution, as radial trends are quite sensitive to sky subtraction problems. 


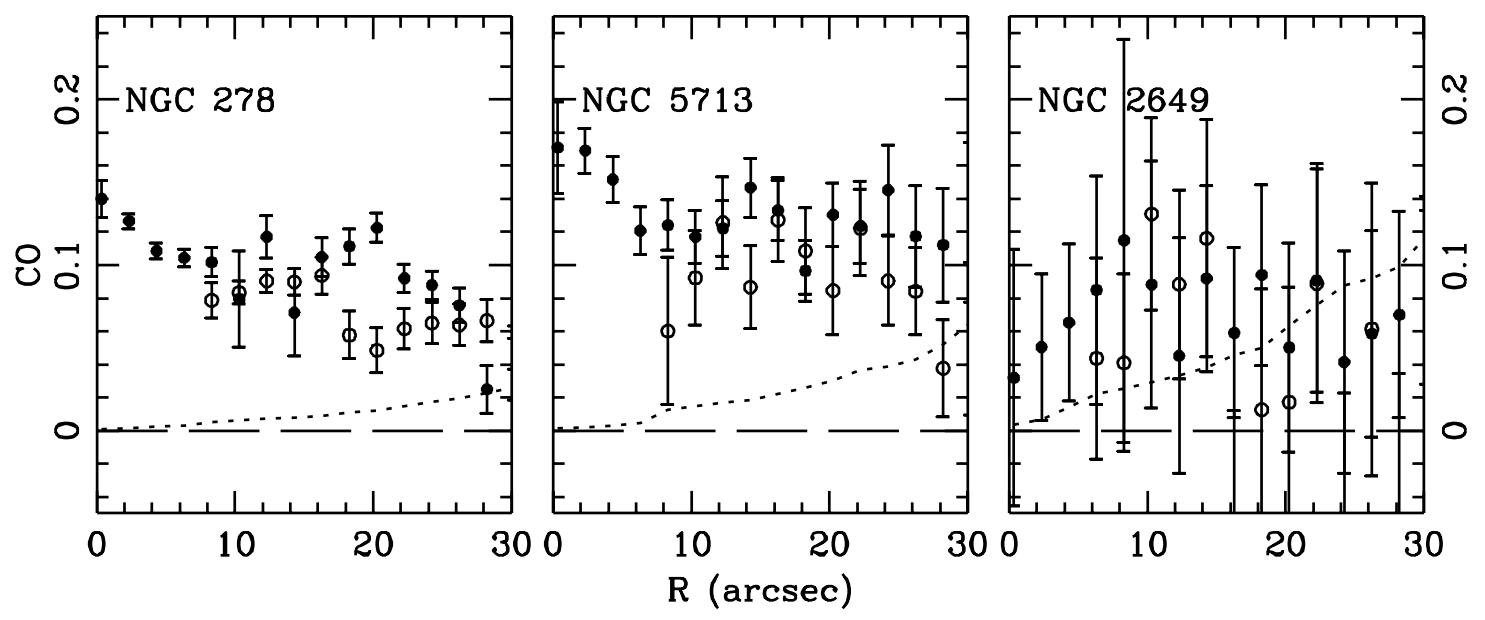

Fig. 2.- CO index profiles for the sample galaxies, corrected for redshift effects. Solid points represent the $\mathrm{CO}$ index of bright regions; open points represent the $\mathrm{CO}$ index of background regions. Error bars on the individual points represent random errors due to photon statistics. The dotted curve represents the estimated sky subtraction error in the $\mathrm{CO}$ index zero point as a function of radius. At radii where both bright and background region $\mathrm{CO}$ indices are defined, the sky subtraction error plotted is for background regions; that for bright regions is typically smaller by a factor of $1.5-2$. The dashed curve marks the $x$-axis for reference. Note that the difference between the $\mathrm{CO}$ indices of bright and background regions is less affected by sky subtraction errors than is the $\mathrm{CO}$ index zero point. 
NGC 5713 also shows a central peak in $\mathrm{CO}$ absorption at $\mathrm{CO} \approx 0.17$. At larger radii there are weak variations in $\mathrm{CO}$ index. The index of bright patches lies generally above that for faint regions from $8^{\prime \prime}$ to $30^{\prime \prime}$, but with significance rarely exceeding $1 \sigma$. The background disk has an index $\mathrm{CO} \approx 0.10$ in the inner regions. The "bright region" mask in this galaxy basically follows the bulge, bar, and single bright spiral arm as galactocentric radius increases. The gradient in $\mathrm{CO}$ index near the center could be due to either nuclear star formation activity or a metallicity gradient.

NGC 2649 shows no significant variations in CO index between regions or between nucleus and disk. While this galaxy has the lowest surface brightness in the sample and therefore modest signal to noise ratio, arm-interarm differences $\Delta \mathrm{CO} \gtrsim 0.10$ can be ruled out.

\subsection{Overall $\mathrm{CO}$ indices}

The overall CO indices measured for our sample are generally below those of elliptical and S0 galaxies published by Frogel et al (1978). We have examined the expected evolution of $\mathrm{CO}$ index with time for a composite stellar population, using a modified version of Bruzual and Charlot's GISSEL spectral synthesis package to produce CO index predictions for solar metallicity and various star formation histories. These predictions will underestimate the $\mathrm{CO}$ index for periods when supergiants dominate the near-IR light, because the spectral libraries used were incomplete for M supergiants (Bruzual \& Charlot 1993) and M giant spectra were used instead. However, for older stellar populations (age $t \gtrsim 10^{8.5}$ years) the results should be fairly accurate. These results (shown in figure 3) show only a weak dependence of $\mathrm{CO}$ index on population age for a galaxy with continuous star formation ongoing for $t \geq 10^{8.5} \mathrm{yr}$, with an overall slight increase in CO index from $10^{8.5} \mathrm{yr}$ to $10^{10.3} \mathrm{yr}$. The CO index for an instantaneous burst fluctuates considerably more but the overall trend is again a weak increase from $10^{8.5} \mathrm{yr}$ to $10^{10.3} \mathrm{yr}$. The model index for a typical elliptical galaxy population (a single burst $\sim 10^{10} \mathrm{yr}$ old) is $\approx 0.16$, while that for a stellar disk (approximated by continuous star formation over $10^{9}-10^{10} \mathrm{yr}$ ) is $\approx 0.13-0.14$. Corrections for the approximation of red supergiant spectra by red giant spectra are unlikely to increase the continuous star formation CO index by more than $\sim 0.04$ at $10^{9} \mathrm{yr}$ or by $\sim 0.02$ at $10^{10} \mathrm{yr}$ (see figures 8-9 of Charlot \& Bruzual 1991, and tables 2-3 of Bell \& Briley 1991). Thus, regions of a disk galaxy where the star formation rate has not been unusually high in the preceding $10^{9} \mathrm{yr}$ need not have a $\mathrm{CO}$ index exceeding that for an elliptical galaxy population.

It is also possible that the comparison of our CO indices with those of Frogel et al 


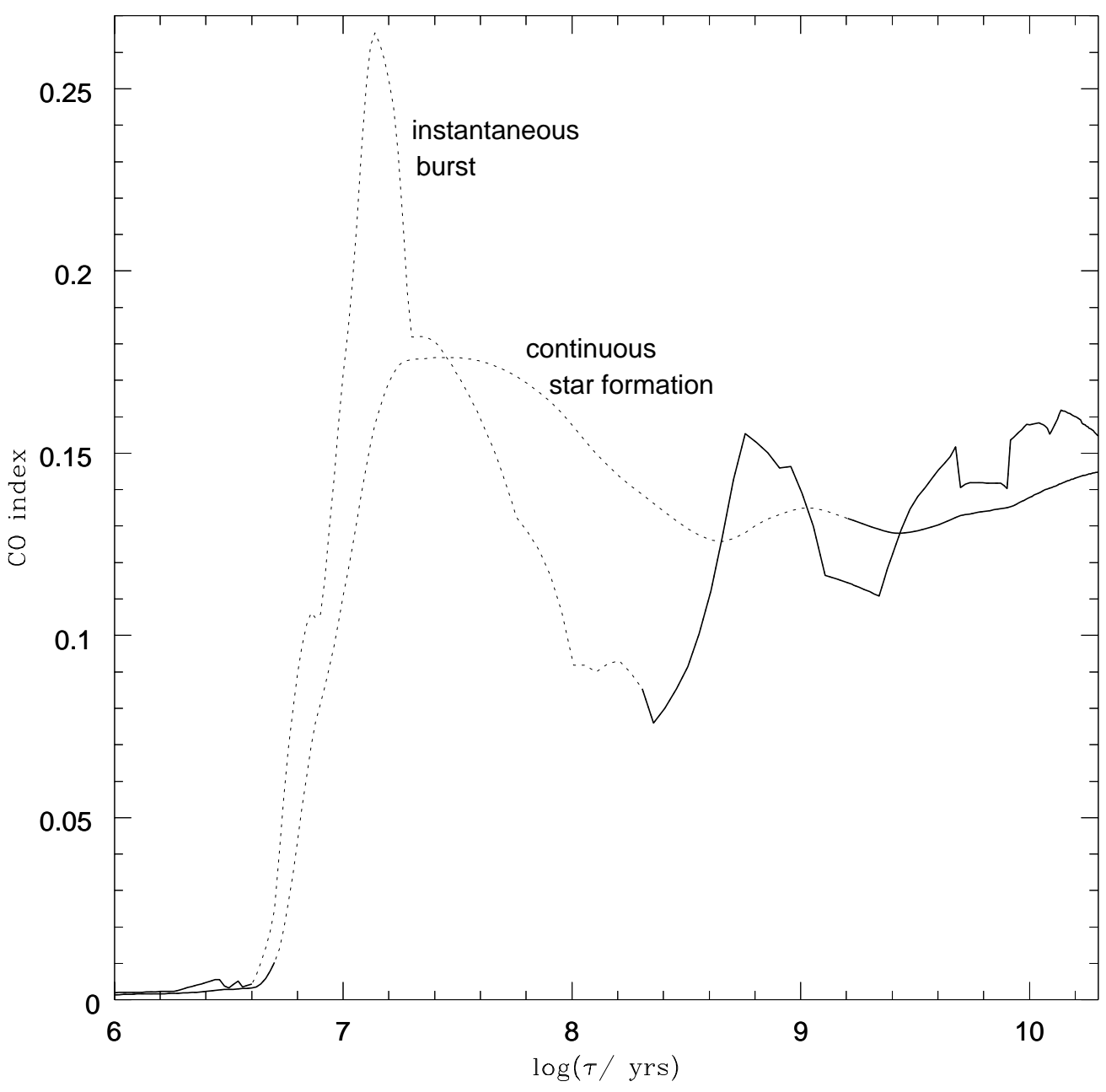

Fig. 3.- CO index as a function of stellar population age, based on a spectral population synthesis code ("GISSEL") by Bruzual \& Charlot (1993). Curves for an instantaneous burst of star formation and for continuous star formation at a fixed rate are shown. We assumed a Scalo (1986) initial mass function with cutoffs at $0.1 M_{\odot}$ and $65 M_{\odot}$. For regimes where red supergiants dominate the $\mathrm{K}$ band light, the model indices will be underestimated, because spectrophotometric libraries for red supergiants were not complete when the code was written, and $\mathrm{M}$ giant spectra are used to represent the light of $\mathrm{M}$ supergiants. The CO index curves are dotted where supergiants contribute over half of the total $\mathrm{K}$ band light, and solid elsewhere. (The supergiant contribution was estimated from figures 8 and 9 of Charlot \& Bruzual 1991.) CO indices were computed by convolving model spectra with the Apache Point Observatory $2.21 \mu \mathrm{m}$ and $2.34 \mu \mathrm{m}$ filters; the zero point of the resulting CO indices was set to give zero CO index to the model spectrum for Vega. 
(1978) are affected by systematic differences between the data sets. One such difference could be in the filter system. The zero point of our $\mathrm{CO}$ indices is constrained to match that of Frogel et al by our choice of standard stars from the list of Elias et al (1982). However, the standard stars we observed all had small CO indices, so that a multiplicative rescaling of our indices may be required to bring them into agreement with the system of Frogel et al (1978). Differences in our K correction methods may also have a small effect intercomparison of the indices.

In any case, modest uncertainties in the absolute calibration of our $\mathrm{CO}$ indices have comparatively minor effects on our analysis, which is based primarily on relative CO indices between different regions of disk galaxies.

\subsection{The fractional contribution of a young population}

We now wish to interpret spatial variations in the $\mathrm{CO}$ index in terms of changes in stellar populations. Consider a background stellar population having CO index $B$, and a bright region with ongoing star formation having index $S$. Suppose that the difference in $\mathrm{CO}$ index is due entirely to the presence of the young stellar population, and that this population has CO index $Y$. Together, $B, Y$, and $S$ determine the fractions $x_{c}$ and $x_{b}$ of the 2.21 and $2.34 \mu \mathrm{m}$ light in the star forming region that must be due to the young stellar population. The relevant expressions (derived below) are

$$
x_{c}=\frac{10^{0.4(S-B)}-1}{10^{0.4(S-B)}-10^{0.4(S-Y)}} \quad \text { and } \quad x_{b}=10^{0.4(S-Y)} x_{c}=\frac{10^{0.4(S-B)}-1}{10^{0.4(Y-B)}-1} .
$$

Assuming that the young population has metallicity at least that of the older populations, and that its age is $\sim 10^{7}$ years so that red supergiants are present, we expect $Y>B$. $S$ will be intermediate between $B$ and $Y$, because it is the index for a mixture of the background population and the young population. Under these conditions, the above relations may be approximated by

$$
x_{c} \approx x_{b} \approx(S-B) /(Y-B)
$$

To derive these results, we introduce auxiliary quantities $c_{B}, c_{S}, c_{Y}, b_{B}, b_{S}, b_{Y}$, where $c_{i}$, $b_{i}$ represent the flux densities from the different stellar populations in the bright patch at the continuum $(2.21 \mu \mathrm{m})$ and band head $(2.34 \mu \mathrm{m})$ filter bandpasses. The subscripts ${ }_{B}, Y$, and ${ }_{S}$ label the contributions from the background, young, and total populations respectively, so that $c_{S}=c_{B}+c_{Y}$ and $b_{S}=b_{B}+b_{Y}$. By construction, $2.5 \log _{10}\left(c_{B} / b_{B}\right)+q=B$, so that $\left(c_{B} / b_{B}\right)=10^{0.4(B-q)}$. Similarly, $\left(c_{Y} / b_{Y}\right)=10^{0.4(Y-q)}$ and $\left(c_{S} / b_{S}\right)=10^{0.4(S-q)}$. The fractional contributions of the young stellar population to $c_{S}$ and $b_{S}$ are then $x_{c}=c_{Y} / c_{S}$ 
and $x_{b}=b_{Y} / b_{S}$. Dividing these gives

$$
\frac{x_{b}}{x_{c}}=\frac{b_{Y}}{c_{Y}} \frac{c_{S}}{b_{S}}=10^{0.4(S-Y)},
$$

and $x_{b}=10^{0.4(S-Y)} x_{c}$ as stated above. The fractional contributions of the background population to $c_{S}$ and $b_{S}$ are $1-x_{c}=c_{B} / c_{S}$ and $1-x_{b}=b_{B} / b_{S}$. Dividing these gives

$$
\frac{1-x_{b}}{1-x_{c}}=\frac{b_{B}}{c_{B}} \frac{c_{S}}{b_{S}}=10^{0.4(S-B)} .
$$

Substituting $10^{0.4(S-Y)} x_{c}$ for $x_{b}$ in this expression and solving for $x_{c}$ gives the formulae stated above.

The largest tabulated CO indices in the models by Bell and Briley (1991) are $\mathrm{CO} \approx 0.06+0.015[A / H]$ for $4500 K$ dwarfs, $\mathrm{CO} \approx 0.21+0.06[A / H]$ for $4000 K$ giants, and $\mathrm{CO} \approx 0.29+0.08[A / H]$ for $4000 K$ supergiants, where $[A / H]$ is metallicity relative to solar and is unlikely to lie outside the range $0 \gtrsim[A / H] \gtrsim-1$ for the galaxies in our sample. The $\mathrm{CO}$ indices for composite stellar populations can attain values $\sim 0.25$ (e.g., the strongest indices Persson et al [1983] measured for star clusters in the Large Magellanic Cloud were $\mathrm{CO}=0.255$ for NGC 1767, 0.245 for NGC 2002, and 0.24 for NGC 1805). This value is also roughly consistent with the indices that Lançon, Rocca-Volmerange, \& Thuan (1996) find in population synthesis models for starbursts. We will therefore use $\mathrm{CO}=0.25$ as an illustrative value of $Y$ in the following discussion, while reminding the reader that $Y$ depends on the starburst age, initial mass function, and metallicity in a complex fashion.

Substituting in observed values for the bright patches in the NGC 278 ring $\left(15^{\prime \prime} \lesssim r \lesssim 25^{\prime \prime}\right)$, we find that $S \approx 0.12$, while in the background regions at the same radius $B \approx 0.06$. Taking $Y=0.25$, we then find $x_{c} \approx 0.33$, i.e., one third of the light must come from the extra stellar population. This is consistent with the surface brightness increase in the bright patches, which are a factor $\sim 1.45$ above the background regions in the annulus $15^{\prime \prime} \lesssim r \lesssim 25^{\prime \prime}$.

In NGC 5713, the nuclear CO index (0.17) exceeds that in the nearest background region $(\sim 0.10)$. If this is due to nuclear star formation, our model with $S=0.17, B=0.10$, and $Y=0.25$ gives $x_{c} \approx 0.48$, so the extra stellar population would have to contribute about half the light. The nuclear surface brightness exceeds that of the disk at $r=10^{\prime \prime}$ by a magnitude, so there is no difficulty here in "hiding" the flux from an additional population. However, a young stellar population is not a unique explanation in this case, because the metallicity may differ between the nucleus and the comparison region, which is $\gtrsim 1 \mathrm{kpc}$ away. 
For NGC 2649, the typical background disk has a low CO index, $B \sim 0.06$. The upper limit to $\mathrm{CO}$ index variations corresponds to $S-B \lesssim 0.10$ for $r<25^{\prime \prime}$. Hence, we find $x \lesssim 0.5$, i.e., the surface brightness is probably dominated by older stars, although we cannot rule out a very substantial contribution from young stars in this relatively low surface brightness system.

\subsection{Hypothesis testing}

We can consider our results in terms of two extreme null hypotheses: First, that NIR light faithfully traces mass; and second, that the departures from some model are due entirely to the presence of star formation activity. The first implies that the CO index should not vary azimuthally, while such variations are expected in the second and can be modeled subject to certain assumptions.

Because the mass to light ratio of a stellar population is sensitive to both age and metallicity, our first hypothesis in general implies a spatially invariant CO index. In practice, the dependence of the $\mathrm{K}$ band mass to light ratio on metallicity is weak (e.g., Kodama \& Arimoto 1997), so that a metallicity change of 1 dex can change the CO index in an old population by $\sim 0.04$ (Bell \& Briley 1991) and the $\mathrm{K}$ band mass to light ratio by $\sim 20 \%$. Thus, a radial gradient in the CO index could be consistent with only a modest change in mass to light ratio. However, substantial azimuthal gradients in metallicity are not expected in the disk component of a galaxy, because phase mixing will suppress them within a few orbital times. Thus, azimuthal variations in CO index are best explained by variations in the age structure of the stellar population. The test of our first null hypothesis is then to look for such azimuthal variation. NGC 278 clearly fails this test, while NGC 2649 and NGC 5713 pass given the uncertainties in the observations.

Producing a detailed prediction of the CO index as a function of radius under the second hypothesis requires assumptions about the stellar populations. We prefer instead the more general model fitting approach described in the following section.

\section{4. $\quad$ Model fitting}

In addition to null hypothesis testing, we can treat the difference $\Delta \mathrm{CO}$ between the $\mathrm{CO}$ indices of background and bright regions as a free parameter and determine its value through $\chi^{2}$ minimization. The null hypotheses adopted above then become special cases of this more general model with $\Delta \mathrm{CO}=0$ and $\Delta \mathrm{CO} \approx 0.1-0.15$ respectively. We 
have studied this model for several sets of CO index measurements, corresponding to different regions of the sample galaxies. The first of these is the bar region in NGC 5713, defined as $8^{\prime \prime} \leq r \leq 25^{\prime \prime}$, where we find $\Delta \mathrm{CO}=0.035 \pm 0.010[ \pm 0.020]$. (Here the first error bar is derived from the photometric uncertainties in the data, while the second is derived from bootstrap resampling of the input CO index measurements. The second error estimate thus represents the uncertainty caused by spatial variations in $\Delta \mathrm{CO}$.) The second region tested was the grand design spiral in NGC 2649 (all data with $r<27^{\prime \prime}$ ), with $\Delta \mathrm{CO}=0.052 \pm 0.037[ \pm 0.053]$. The "ring" region of NGC $278\left(9^{\prime \prime}<r<30^{\prime \prime}\right)$ shows $\Delta \mathrm{CO}=0.062 \pm 0.011[ \pm 0.038]$. It thus appears that there is a marginal $\mathrm{CO}$ index excess in the bar of NGC 5713, and a significant excess in the ring in NGC 278 (although this absorption is itself patchy, leading to a large error estimate from bootstrap resampling). There could be absorption in NGC 2649 but the data are not sensitive enough to make a definitive statement. The best fitting $\Delta \mathrm{CO}$ yields $\chi^{2}$ per degree of freedom of 1.4 for NGC 2649, 2.7 for NGC 5713, and 4.8 for NGC 278. This is quantitative evidence that the model is an oversimplification, which is not surprising considering that variations in star formation history and metallicity can result in a spatially variable $\Delta \mathrm{CO}$.

\subsection{The contribution of young populations to total luminosity}

We can estimate the contribution of red supergiants to the total NIR light from the sample galaxies. For NGC 278, the bright regions in the two annuli with significant CO index deviations account for total flux corresponding to $K^{\prime}=10.48$, or $9 \%$ of the galaxy's total flux. As shown above, $\sim$ one third of this is from a young stellar population, so young stars contribute not less than $\sim 3 \%$ of the galaxy's total $2 \mu \mathrm{m}$ emission. In NGC 2649 , the bright region masks (here corresponding well to the nucleus and spiral arm ridges) account for $53 \%$ of the total light, so no more than $1 / 4$ of the light can be from a young stellar population.

Thus, while young stars do not dominate the global NIR flux from disk galaxies, they can be locally quite important in star forming regions. This can bias arm:interarm amplitudes and similar measures of galactic substructure.

\subsection{Aside: The distance to NGC 2649}

We can use our data together with published gas kinematics to estimate the distance to NGC 2649 using the K band Tully-Fisher relation (Malhotra, Spergel, Rhoads, \& Li 
1996). The inclination angle of NGC 2649 can be estimated by assuming that a logarithmic spiral describes its arm geometry. We found inclination angle $i=30^{\circ} \pm 3^{\circ}$. This can be used to infer a deprojected line width. The observed HI linewidth is $\sim 260 \mathrm{~km} / \mathrm{s}$ at $20 \%$ of peak (Lewis 1987, quoted in Huchtmeier \& Richter 1989). The corrected width is then $\Delta V=520 \pm 50 \mathrm{~km} / \mathrm{s}$, somewhat exceeding the Milky Way's line width (cf. Malhotra et al 1996) and implying an absolute $\mathrm{K}$ band magnitude $M_{K}=-24.0 \pm 0.4$. The dominant uncertainty is from the inclination correction. The resulting distance is $d=66 \pm 13 \mathrm{Mpc}$ (corresponding to $H_{0}=64 \pm 13 \mathrm{~km} / \mathrm{s} / \mathrm{Mpc}$ ). At this distance the inferred scale length in our K band data is $5 \pm 1 \mathrm{kpc}$, larger than the Milky Way (cf. Spergel, Malhotra, \& Blitz 1996) and consistent with NGC 2649's larger rotation speed.

\section{Discussion}

We now wish to consider our results in the context of issues raised in the introduction.

Interpreting measured $\mathrm{CO}$ indices in terms of the ages of stellar populations is complicated by the variety of factors that can affect $\mathrm{CO}$ absorption strength. For individual stars, these factors are the gravity, temperature, and metallicity at the photosphere. These factors in turn are primarily determined by the mass, initial composition, and age of a star, though details of the star's history (for example, binary interactions) may be important for some stars. For a composite stellar population, the distribution of mass, composition, and age can be derived from the initial mass function, chemical evolution history, and star formation history of the galaxy. In principle, then, we can determine the $\mathrm{CO}$ index expected from a given galactic evolutionary history, whence we can learn about the contribution of recently formed stars to $2 \mu m$ light.

In practice, the steps linking a model evolutionary history to an inferred $\mathrm{CO}$ index encompass several uncertainties. First, the problem is underconstrained, so that additional information must be used. In practice this will entail assuming that the initial mass function (IMF) does not vary with time and takes a form that fits observations of nearby galaxies (e.g., Scalo 1986). Second, the details of converting stellar age, metallicity, and mass to a CO index are not well known. Empirical approaches to this problem associate libraries of observed stellar spectra with the controlling physical parameters. A shortage of well measured supergiant spectra in current libraries led Bruzual and Charlot (1993) to use M giant spectra for M supergiants. This will lead to an underestimate of the supergiant contribution to the $\mathrm{CO}$ index. The best available tables of theoretical CO indices (Bell \& Briley 1991) do not extend to effective temperatures $T_{\text {eff }}<4000 \mathrm{~K}$, and so do not offer a complete solution to this difficulty. Third, the late evolution of massive stars is 
complicated by mass loss and convective overshooting phenomena, and is thus difficult to predict on theoretical grounds (Langer \& Maeder 1995). This means that the distribution of stellar age, mass, and metallicity can only approximately be mapped into a distribution of luminosity and effective temperature for the young, massive stars we are concerned with.

Much careful work will be necessary to reduce the uncertainties in population synthesis models of the CO index. Many of the difficulties can however be ignored if we study directly measured $\mathrm{CO}$ indices of star clusters of known age. In particular, the purely theoretical difficulties of determining which stars become red supergiants and how long they live in that phase go away. Likewise, any incompleteness in spectral libraries (whether theoretical or empirical) becomes irrelevant. Accurate age estimates for the observed clusters become the main difficulty. The uncertainties here are smaller if the cluster ages are derived from main sequence fitting, because we then depend on our understanding of main sequence stellar evolution, which is more certain than our understanding of supergiant phases of massive stars.

In figure 4, we plot CO indices (measured by Persson et al 1983) against age estimates (by Elson \& Fall 1985, 1988) for star clusters in the Large Magellanic Cloud (LMC). Elson and Fall used main sequence fitting to estimate some cluster ages and to calibrate an age sequence in a two-color diagram; ages for the remaining clusters were determined from their positions along this sequence. The resulting CO-age relation shows a statistically significant correlation with a large scatter. Besides the observational errors in CO index and age, this scatter includes contributions from Poisson fluctuations in the comparatively small number of evolved stars (especially in the younger clusters; see Santos \& Frogel 1997) and from possible metallicity variations among clusters. Metallicity differences between and within galaxies may also exist of course. Comparing the CO indices for NGC 278 to those for LMC clusters yields a characteristic age $\sim 10^{8}$ years for the regions of strongest $\mathrm{CO}$ absorption, and $\sim 10^{9}$ years for the rest of the disk. In reality, of course, the CO index for diffuse light from a galactic disk encompasses stars of a wide range of ages. It is nonetheless reassuring that the bright regions show a characteristic age comparable to a typical galactic rotation time, while the background disk appears much older than a rotation time. Similarly, the measured CO indices for NGC 2649 and for the background regions of NGC 5713 correspond to ages in excess of galactic rotation periods.

Our conclusions regarding the fraction of total NIR light that comes from young stars are consistent with the small observed scatter in the $2 \mu \mathrm{m}$ Tully-Fisher relation $(<0.45 \mathrm{mag}$ at the $95 \%$ level [Malhotra et al 1996]). If star formation rates differ widely among galaxies of the same rotation speed, the scatter in the Tully-Fisher relation cannot be smaller than the scatter in the fraction of light from young stars. 


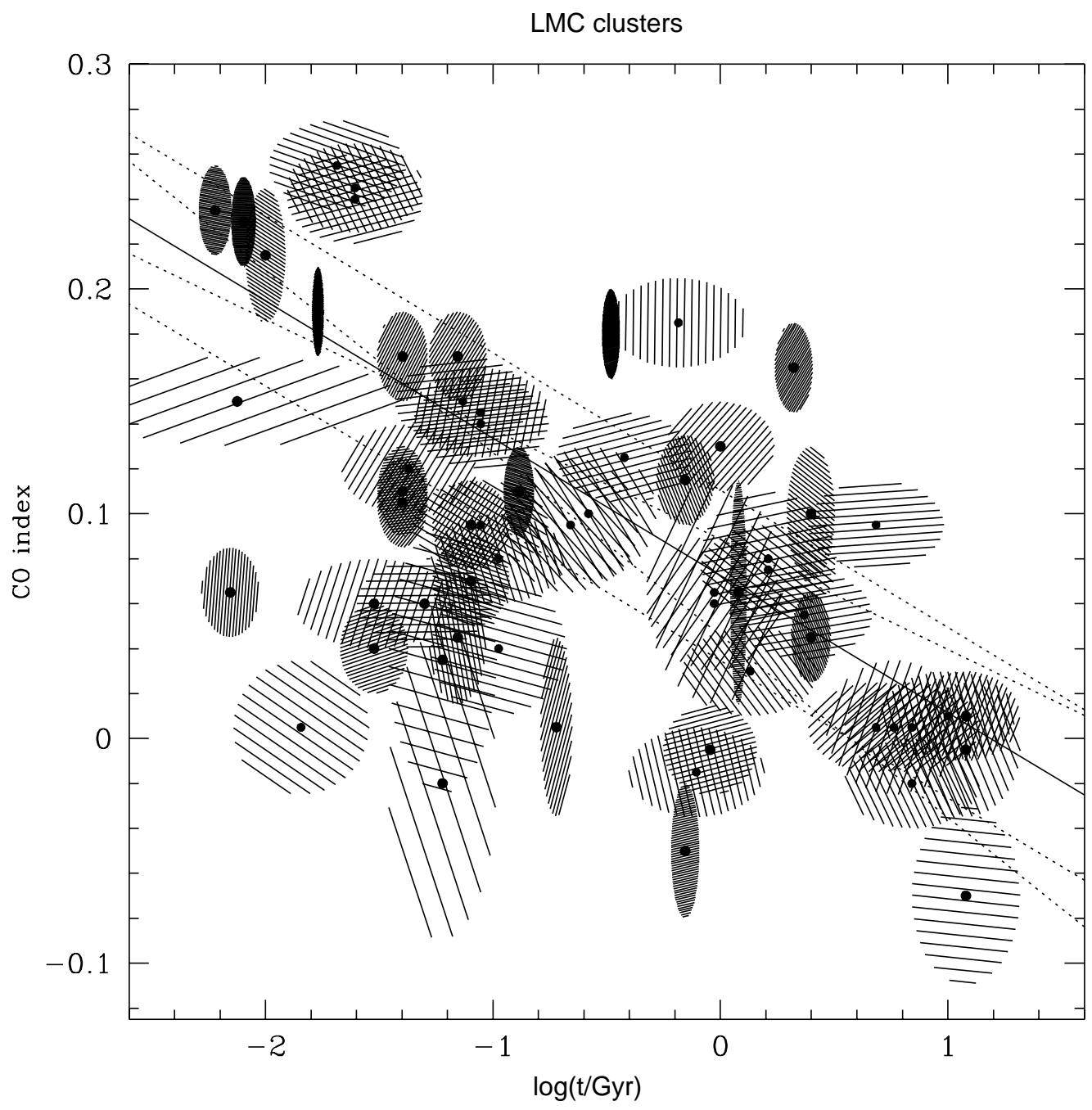

Fig. 4. - CO index as a function of age for star clusters in the Large Magellanic Cloud. 60 clusters are shown. Photometry is from Persson et al (1983) and age estimates are from Elson \& Fall $(1985,1988)$. The crosshatched regions are the $1 \sigma$ error ellipses for the clusters. Also shown are lines for the least squares fit (solid; based on the 2D error ellipses shown) and for $1 \sigma$ changes in the fitted line intercept and/or slope (dotted). 
Other indicators of star formation activity could also be used to estimate the contribution of young stars to near-infrared light. Each involves its own set of uncertainties. The CO bandhead is a direct test for supergiant light in the $2 \mu \mathrm{m}$ band. Other common diagnostics trace either the presence of ultraviolet (UV) radiation from hot, young stars, reprocessed either by gas (as recombination lines) or by dust (as mid- and far-infrared emission); or the byproducts of supernova shocks, reprocessed as synchrotron radiation. The absence of such tracers could demonstrate that features in near-infrared light are due to old stars. However, their presence would not unambiguously prove that NIR features are due to young stars, because of the astrophysical uncertainties in determining the number of red supergiants that evolve from a given population of young main sequence stars.

\section{Conclusions}

We have measured the strength of the $2.3 \mu \mathrm{m}$ CO absorption bands in three nearby disk galaxies. The sample covers a substantial range of morphologies and global star formation rates, but does not include any starburst galaxies. We find that the CO index shows significant spatial variations in the brightest regions of our two best-measured galaxies. The variations are consistent with $\sim 1 / 3$ of the light from these regions being produced by a young stellar population whose near-infrared light is dominated by red supergiant stars. The characteristic age of such a population, $\sim 10^{7}$ years, is smaller than galactic rotation periods, so that these stars must still be near their birthplaces. Globally, the contribution of such stars to the galaxies' near-IR luminosity is a few percent. Thus, while the overall $2 \mu m$ emission is primarily due to old stellar populations, local features in the near-infrared light of disk galaxies can be due primarily to young stars.

I thank David Spergel, Jim Gunn, Michael Strauss, Sangeeta Malhotra, Ed Fitzpatrick, Bohdan Paczyński, Stephane Charlot, Neil Tyson, Hans-Walter Rix, and Jay Frogel (the referee) for many helpful discussions during the course of this work. Thanks also to Roger Bell, who kindly provided the model spectrum used to calculate redshift corrections to the CO index. I am also grateful to the Infrared Processing and Analysis Center for its hospitality. This research has been supported by NSF grant AST 91-17388, NASA grant ADP NAG5-269, NSF traineeship DGE-9354937, and a Kitt Peak National Observatory postdoctoral fellowship. This research has made use of the Simbad database, operated at CDS, Strasbourg, France. This research has made use of the NASA/IPAC Extragalactic Database (NED) which is operated by the Jet Propulsion Laboratory, California Institute of Technology, under contract with the National Aeronautics and Space Administration. 


\section{REFERENCES}

Athanassoula, E. 1996, in Spiral Galaxies in the Near-IR, eds. D. Minniti and H.-W. Rix, Berlin: Springer-Verlag, 147.

Baldwin, J. R., Frogel, J. A., \& Persson, S. E. 1973, ApJ 184, 427.

Bell, R.A. \& Briley, M.M. 1991, AJ 102, 763

Bell, R. A., \& Tripicco, M. J. 1991, AJ 102, 777

Bruzual A., G., \& Charlot, S. 1993, ApJ 405, 538

Casali, M., \& Hawarden, T. 1992, UKIRT/JCMT newsletter, August 1992, p. 33

Charlot, S. \& Bruzual A., G. 1991, ApJ 367, 126

de Vaucouleurs, G., de Vaucouleurs, A., Corwin, H. G., Buta, R.J., Paturel, G., \& Fouqué, P. 1991, Third Reference Catalog of Bright Galaxies, New York: Springer-Verlag (RC3)

Elias, J.H., Frogel, J.A., Matthews, K., and Neugebauer, G. 1982 AJ 87, 1029

Elson, R.A.W. \& Fall, S.M., 1985, ApJ 299, 211

Elson, R.A.W. \& Fall, S.M., 1988, AJ 96, 1383

Frogel, J. A., Persson, S. E., Matthews, K., \& Aaronson, M. 1978, ApJ 220, 75

Frogel, J. A., Cohen, J. G., \& Persson, S. E. 1983, ApJ 275, 773

Grosb $\varnothing l$, P., \& Patsis, P. 1996, in Spiral Galaxies in the Near-IR, eds. D. Minniti and H.-W. Rix, Berlin: Springer-Verlag, 174.

Huchtmeier, W. K., \& Richter, O.-G. 1989, A General Catalog of HI Observations of Galaxies, New York: Springer-Verlag

Kodama, T., \& Arimoto, N. 1997, A\&A 320, 41

Lançon, A., Rocca-Volmerange, B., \& Thuan, T. X. 1996, A\&AS 115, 253

Langer, N., \& Maeder, A. 1995, A\&A 295, 685

Lewis, B. M. 1987, ApJS 63, 515

Malhotra, S., Spergel, D. N., Rhoads, J. E., \& Li, J. 1996, ApJ 473, 687

McWilliam, A., \& Lambert, D. L. 1984, PASP 96, 882

Peletier, R. F., \& Balcells, M. 1996, in Spiral Galaxies in the Near-IR, eds. D. Minniti and H.-W. Rix, Berlin: Springer-Verlag, 48.

Persson, S. E., Cohen, J. G., Matthews, K., Frogel, J. A., Aaronson, M. 1983, ApJ 266, 105 
Press, W. H., Teukolsky, S. A., Vetterling, W. T., \& Flannery, B. P. 1992, Numerical Recipes, 2nd edition, New York: Cambridge University Press.

Quillen, A. C. 1996, in Spiral Galaxies in the Near-IR, eds. D. Minniti and H.-W. Rix, Berlin: Springer-Verlag, 157

Robin, A., Crezé, M., \& Mohan, V., 1992 ApJ 400, L25

Rix \& Rieke 1993, ApJ 418, 123

Rix, H.-W., \& Zaritsky, D. 1995 ApJ 447, 82 [RZ]

Santos Jr., J. F. C., \& Frogel, J. A. 1997, ApJ 479, 764.

Sandage, A., \& Bedke, J. 1994, The Carnegie Atlas of Galaxies, Carnegie Institute of Washington publication 638

Scalo, J. M. 1986, Fundamentals of Cosmic Physics, 11, 1

Schaller, G., Schaerer, D., Meynet, G., \& Maeder, A. 1992, A\&AS 96, 269

Spergel, D.N., Malhotra, S., \& Blitz, L. 1996, in Spiral Galaxies in the Near-IR, eds. D. Minniti and H.-W. Rix, Berlin: Springer-Verlag, 128

Terndrup, D. M., Davies, R. L., Frogel, J. A., DePoy, D. L., \& Wells, L. A. 1994, ApJ 432, 518

Van Buren, D., \& Kong, M. 1997, in preparation

Wainscoat, R. J, \& Cowie, L. L. 1992, AJ 103, 332

Wray, J. D. 1988, Color Atlas of Galaxies, Cambridge: Cambridge University Press 
Fig. 1. - Images of the galaxies in $K^{\prime}$ band $(2.12 \mu m)$ and of the masks used for photometry. Each image is 2.06 arcminutes on a side. North is up, East is left. Images: a. NGC 2649; b. NGC 278; c. NGC 5713. Masks: Black areas are bright region masks, grey areas are background region masks. d. NGC 2649; e. NGC 278; f. NGC 5713. 
This figure "fig1.jpg" is available in "jpg" format from: http://arxiv.org/ps/astro-ph/9710189v1 